\title{
LUCHA ANTITUBERCULOSA EN NORUEGA SUECIA Y DINAMARCA
}

\author{
Por la Dra. Gabriela CIFUentes heríera (*) \\ Hospital Luis Calvo Mackenna.
}

Es conocido de todos el problema que crea la tuberculosis tanto en el terreno médico como en el social, y la enorme importancia que tiene el considerarla bajo este doble aspecto, se bace más notoria cuando nos enfrentamos con la labor desarrollada por los paises nórélicos en la lucha contra este flagelo, lo que tuve la felicidad de poder comprobar en mi reciente visita a esos países.

Cerca de 30 años de empeñoss y estrecha colaboración de parte del médico, de la sociedad y del Estado mismo. han dado por resultado una disminución considerable de la mortalidad por tuberculosis en estos países. Las cifras estadísticas siguientes nos revelan la efectividad de esta campaña:

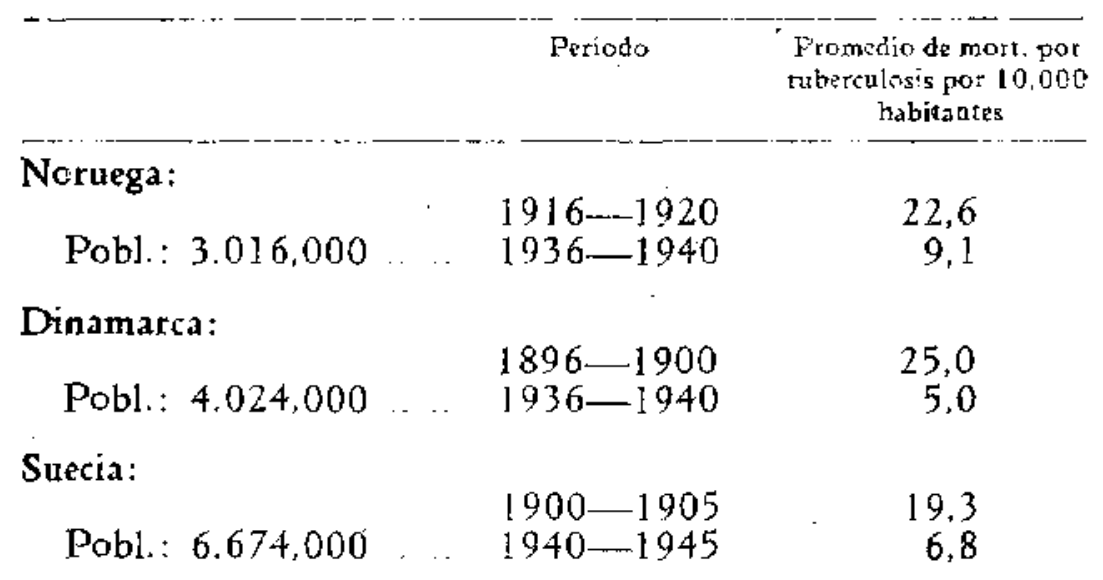

(*) En viajc de estudio jor Succia, Dinamarca y Noragga en 1946 , comisionada por los Servicios de Beneficencia. Protección de la lnfancia y Bienestar del Ejército. 
tan bajos?

¿Qué es lo que se ha hecho para lograr estos promedios

Se puede decir a grandes rasgos, que dos han sido los factores que se han puesto en juego correlacionado para lograrlo:

1. La profilaxia pasiva o indirecta, si así pudiera ilamarse a la que va dirigida a pesquisar los casos, separarlos del medio, etc., $y$

2. La profilaxis activa o directa, que se hace por medic de la vacunación.

\section{PROFILAXIA PASIVA}

Esta se hace mediante: declaración cbligatoria, desinfección, central de tuberculosis y dispensarios. Liga Nacional de lucha antituberculosa, ayuda de los Municipios y del Estado. horpitales y sanatorios, establecimientos para los niños de familias tuberculosas, control del ganado y personal de lecherias.

\section{Declaración obligatoria.}

Considerada la tuberculosis dentro del terreno de las enfermedades contagiosas, a través de muchos ensayos $y$ discusicnes, se ha logrado establecer por disposiciones legales, que datan de largo tiempo, que sea como todas ellas una enfermedad de "declaración obligatoria".

Toda persona que sepa de la existencia de un caso de tuberculosis, que no haya sido deciarado, y los médicos que comprueben un caso, o que certifiquen la defunción de una persona por esta enfermedad, deben dar un informe por escrito a un "Comité de Salud Pública", perteneciente al Municipio, el que a su vez. lo comunica al Dispensario Central de Tuberculosis, situado en la capital.

\section{Desinfección.}

Comprobado el caso. el Comité de Salud Publica intervienc inmediatamente mediante el "servicio gratuito de desinfección" en el hogar afectado. 


\section{Central de tubercnlosis $y$ dispensarios.}

La colaboración de los Municipios en la labor de mantenimiento de la higiene y salud pública es enorme. Frente al problema de la tuberculosis, éstos actúan a través de la Central de Tuberculosis y los Dispensarios.

La Central de Tuberculosis o Dispensario Central, situado en la capital junto con los demás Dispensarios, repartidos en todo el país, realizan una labor de previsión y en ningún momento toman a su cargo tratamientos. Su campo de acción abarca: el examen médico, las tuberculino-reacciones, los exámenes de investígación de laboratorio (sedimentación, desgarro, lavado gástrico, cultivo, orina), radioscopías y radicgrafías (corrientes y de grupo).

En algunas ocasiones, los Dispensarics emplean su propio laboratorio de investigación $y$, en otras, las muestras son enviadas al Laboratorio Bacteriológico del Estado. En Dinamarca, por ejemplo, el Dispensario Central de Copenhague, envía las muestras de desgarro y las muestras para cultivo, al Instituto del Suero. El envío se hace per correo ordinario en dispesitivos especiales y en sobres libres de franqueo.

La persona que llega a un Dispensario par'a ser controlada, debe ser enviada por un médico particular. o de hospital, sanatorio, fábrica o escuela.

Cuando un médico particular descubre o sospecha un caso de tuberculosis, junto con hacer la declaración al Comité de Salud Pública, tiene la obligación de enviat al enfermo al dispensatio, para que sea controlado y fichado. Los resultados de los exámenes son remitidos al médico que lo envió con la firma del colega que ha efectuado el control. En el caso de tratarse de una tuberculosis confirmada, el dispensario debe ser informado sobre el destino del paciente y de los cuidados y controles que recibe.

Descubierto un caso de tuberculosis, el dispensario se encarga de controlar el medio que rodeaba al enfermo; esto lo hace por intermedio de las enfermeras sanitarias, quienes se encargan de citar a todas las personas que viven en las cercanías del foco para controles de tuberculina, sedimentación. exámenes de rayos, etc.

Tanto los dispensarios centrales como los demás, poseen equipos para la atención completa individual y colectiva (obreros, escolares, oficinistas, etc.). Este ccntrol es obligatorio y extensivo a su propio personal, dos veces al año.

Para el logro de estos propósitos, los dispensarios poseen personal médico, enfermeras, enfermeras sanitarias que visitan 
los hogares, radiólogos y laboratcristas, además del personal administrativo $y$ de estadística. Todos trabajan 8 horas diarias. Los médicos trabajar de 3 a 4 horas para el público. en dos equipos, uno en la mañana y otro en la tarde. El médico jefe permanece toda la jornada y asiste con su personal médico a reuniones radiológicas que se celebran dos veces por semana en ei establecimiento y en las que se discuten los casos dudosos y de mayor interés.

La atención al público, es entcramente gratuita y los dispensatios de todo el pais atienden en igual forma que el Central y deben informar, además, a este último sobre todos los casos atendidos, como así mismo de las defunciones y labor desarrollada.

El dispensario Central, situado en la capital. lleva el contrcl estadístico de todo lo que se ha hecho en $\epsilon 1$ pais con los casos declarados y con los sospechosos de tuberculosis. comprendidos los adultos y los niños, ya que la lakor preventiva de los dispensarios alcanza a todas las edades.

Llama la atención las estadisticas tan cuidadosamente llevadas en estas centrales. En la Central de Copenhague, por ejemplo. existe un sistema de clasificación de los casos par lesiones y exámenes practicados, que permiten una gran uniformidad de método.

Para facilitar la tarea de hospitalización, los dispensarios están en relación directa con algunos hospitales y sanatorios de tuberculosos.

Los dispensarios amplian su labor social, manteniéndose en conexión con algunos Comités Municipales (Ccmité de Salud Pública, Comité del Pobre, por ejemplo) y con la Liga Nacional de Lucha Antituberculosa. Por estos medios logra diferentes beneficios para el público; asi, por ejemplo: la ubicación en "hogares asilo" de los niños con parientes tuberculosos, distribución de cupones para obtener alimentos a precios rebajados, distribución de leche, obtención de subsidios familiares para mejorar las condiciones de alojamiento, desinfecciones, etc.

\section{Liga nacional de lucha antitubercujosa.}

Es una organización que, con carácter privado inició sus labores a principios de este siglo en Noruega, Suecia y Dinamarca. Sus fines estuvieron primero encaminados bacia una labor de propaganda educativa contra la tuberculosis; luego se hizo extensivo a la creación de Dispensatios, Sanato- 
rios y Preventivos Públicos, y atención a los familiares del tuberculcso. Ultimamente ha llegado a tomar parte activa en las vacunacicnes por el B. C. G., para lo cual posee un equipo de médicos y enfermeras.

Para cumplir sus propósitos, la Liga da conferencias, hace exposiciones y se encarga de toda la labor de propaganda en la lucha antituberculosa. Bajo sus auspicios se crearon los primeros dispensarics, de los que ya hemos hablado, y que con el tiempo han ido a la formación de una Central. Por su iniciativa se han construído muchos sanatorios y especialmente hogares asilos destinados a los niños dé familias tuberculosas.

Para el financiamiento de sus diversas actividades. 1a Liga cuenta con el derecho de una emisión especial de estampillas, durante Navidad: con la participaciós de ciertos impuestos, y con un aporte del Estado y de los Municipios.

\section{Ayuda de los Municipios $¥$ del Estado.}

Hemes visto cómo los Municipios contribuyen a la campaña antituberculosa, con los Dispensarios. sus diversos Comités y con su aporte económico a las labores de la Liga Antituberculosa.

El Estado, a su vez, apoya económicamente a los $\mathrm{Mu}$ nicipies y a la Liga Antituberculosa. Además. se ha establecido por ley que. el Estado debe cocoperar con una cierta suma en la construcción de hespitales y canatcrios para tuberculosos. y con otra suma para los gastos de tratamiento y cuidades a los enfermos tuberculosos. En Dinamarca. por ejemplo, el $\mathrm{E}_{3}-$ tado properciona hasta $2.500 \mathrm{kr} .(\$ 15.500)$ por cama, para las construcciones hospitalarias, y paga las $3 / 4$ partes de los gastor que demandan el cuidado y tratamiento de un tuberculoso, la cuarta parte restante es cancelada por "el Seguro $\mathrm{N}_{\mathrm{a}}$ cional for enfermedad", y si el enfermo no es asegurado. la comuna correspendiente se lo paga en f $\approx$ rma de "Socorro Especial".

Debemes hacer notat que existe una preocupación efectisa y evidente en la rebabilitación del tubeicaloso. En este sentido, en Suecia por ejemplo, el Dispensario Central de Estocolmo, posee un departamento especial, donde se estudia y realiza la distribución en los sanatorios de pequeños trabajos manuales remunerados. Fábricas. como la Telefunken Erickson y otras, tienen el compromiso de colaborar, entregando gran cantidad de material para la construcción de aparatos 
de teléfonos, pantallas, choapinos, etc. Esta preocupación de rehabilitar al tuberculoso, no es tan sólo interesante bajo el aspecto económico al dejar de ser este tipo de enfermo un peso muerto en el presupuesto, sino que también tiene un hondo significado de orden moral para el enfermo mismo.

\section{Hospitales y Sanatorios.}

Para la atención del tuberculoso existen numerosos es. tablecimientos variados en su situación geográfica y destinados a distintos tipos de enfermos: asi hay establecimientos a orillas del mar y en las montañas para escrofulosis, tuberculosis ósea, ruberculosis pulmonar y convalecientes.

\begin{tabular}{|c|c|c|c|}
\hline А⿱̃กิ 1943 & Establecimientos & Camas & Hospitalizaciones \\
\hline $\begin{array}{lllllll}\text { Noruega } & . . & . & . . & . & . & . \\
\text { Dinamarca } & . & . & & . & . \\
\text { Suecia } & . & . . & . & . & \ldots & .\end{array}$ & $\begin{array}{r}138 \\
63 \\
101\end{array}$ & $\begin{array}{l}5.725 \\
4,201 \\
8.885\end{array}$ & $\begin{array}{r}6,851 \\
13,052 \\
18,378\end{array}$ \\
\hline
\end{tabular}

Los casos declarados de tuberculosis durante 1943 en Noruega fueron 4,003, y en Dinamarca 3,348. En una población de 3 y 4 millones de hahitantes en estos países, se calcula que existe un promedio de 1.2 cama para tuberculosos por mil habitantes.

Establecimientos para niños de familias tuberculosas.

Existen estabiecimientos especialmente destinados para recibir los niños sanos controlados en el Dispensario y en cuyos hogares se ha descubierto un foco de contagio tuberculoso. La mayoría de estcs asilos-bogares los constituyen fos llamados "hogares de recepción", que funcionan a cargo del "Comité de Bienestar o de Cuidado del Niño", el que se encarga, además, de la colocación familiar, si el caso así Jo requivere.

\section{Control del ganado y personal de lecheria.}

I a fuente de contagio tuberculoso puede provenir de un medio humano o animal, particularmente los bovinos: para esto existe una legislación especial para el control del ganado vacuno, y el personal de lecherías y establos debe someterse a controles médicos frecuentes. 


\section{PROFILAXIS ACTIVA O DLRECTA}

Se hace por la vacunación por el B. C. G.

La vacuna antituberculosa conocida oor B. C. G. (Behring, Calmette, Guerin), consiste en términos generales. en un extracto de bacilos tuberculosos bovinos originariamente virulentos y que tras largos procesos de cultivos en bilis, han pirdido su efecto nocivo sobre el hombre y los animales.

\section{Vias de uso.}

Vix oral, - Inicialmente Calmette preconizaba eí uso de la vacuna por vía oral, pero las pruebas a la tuberculina reveladoras de la alergia, demostraron que ésta era muy lenta en producirse, de modo que se han preferido otras vias de uso.

Vía subcutánea. - Weill-Hallé, en Francia, ha preconizado este método inyectando mayores dosis que las usadas por otras vías, con buenos resultados.

Vía intradérmica. - El Prof. Wallgren, en Suecia, habiendo experimentado con los métodos anteriores, comprobó que las desagradables complicaciones locales son minimas cuando se emplea la vía intradérmica: se emplea la jeringa de Mantoux.

Escarificaciones cutáneas. - Rosenthal, en EE. UU., y Weill-Hallé, en Fráncia, han empleado el método de las escarificaciones cutáneas en forma de cruces (4 cruces de $1 \mathrm{~cm}$. de largo). Birkhaug, en Noruega, entusiasta partidario de este método, ha ideado un dispositivo especial que permite provocar en el área reducida de $2 \mathrm{cms}$. de diámetro, 40 escarificaciones en un solo tiempo.

\section{Dosis.}

Las vacunas son preparadas por el Instituto dei B. C. G. Las dosis que se emplean son variables. En los niños se usa una cantidad que varia de 0,02 a $0.10 \mathrm{mg}$. de B. C. G.

Técnica de escarificación.

Se hace una limpieza con alcohol en la cara externa del brazo o del muslo, o en la región anterior del tórax, cerca de la axila y se deposita en ella, por medio de un cuenta gotas estéril, una porción de la vacuna. Unia vez hecho esto, se procede a la escarificación, procurando que la piel esté 1o 
más extendida posible. Practicada la escarificación, se coJoca un trozo de gasa hidrófila, impregnada con la vacuna y se fija con tela adhesiva.

\section{Principios para la vacunación.}

El Prof. Wallgren, quién es el que ha realizado las mayores experiencias en Escandinavia con las vacunaciones por el B. C. G. en los niños, ha llegado a establecer dos principios fundamentales en su aplicición: de ser infectado.

1. Aplicar la vacuna en todo niño sano, en peligro

2. Proteger al niño vacunado hasta el momento de aparición de la alergia provocada por este método.

$U_{n}$ organismo que cura de una primo-infección tuberculosa, no adquiere más que una relativa inmunidad contra un prcceso secundario o terciarios. La racuna, 31 igual aue la enfermedad, no libera al individuo de estas complicaciones, pero sí lo libera de las formas más graves de tuberculosis primarias.

Las experiencias dei Prof. Wallgren, Scheel, Rinvik, Sahl. Abderson y Belfrage en Escandinavia, las de Weill-Hallé y Turpin en Francia, asi como experiencias practicadas en EE. UU. y Canadá, demuestran que el niño o el adulto vacunado, expuestos en un foco de infección tuberculosia después de aparecida la alergia post-vaccinal, no presentan sino que en ccntadas excepciones focos pulmonares, que no dan sintomas. pero que han sido descubiertos por control a R. X., y gencralmente ban sido personas en las que existía una duda al vacunarlas. que fuera portadora de una infección provocada por germen tuberculoso virulento.

El Prof. Wallgren insiste, que para probar la eficacia de la vacuna no debe tan sólo projederse a la separición del individuo de un foco tuberculcso durante el tiempo mínimo que demora en presentarse la alergia (4 semanas), sino que debe tomarse en cuenta que el individuo que va a ser vacunado tenga sus reaccicnes tuberculinicas completamente negativas.

En Escandinavia se ha llevado al máximo el cumplimiento de estos principios en los niños, y se mantiene a los niños vacunados, aislados en establecimientos especiales. En. los adultos, ha sido más difícil obtener el requisito de aislamiento post-vaccinal; sin embargo, los resultados han sido satisfactorios. 
Al descubrirse un caso de tuberculosis, los niños y demás perscnas expuestas al contagio son examinadas y sometidas a centrol de tuberculina en los Dispensarios.

Los niños que presentan reacción tuberculínica positiva. son sometidos a un control de R. X. y cualquiera que sea el resultado. se procede a enviarles fuera del foco de contagio, a colccación familiar o preventorios. Los que han presentado reacciones tuberculinonegativas son enviados a los establecimientes de "recepción" o de "observación" ya citados, donde se les sigue controlando. Si dentro del lapso de 6 semanas aparecen reacciones tuberculino positivas, son sometidos a control de Rayos, y si no presentan lesiones son devueltos a su propio hogar; si se ha eliminado el foco de contagio, a colocación familiar.o a Preventorio: y si hay lesicnes. son enviados a Sanatorio. Los que dentro del lapso de estas $6 \mathrm{se}$ manas han continuado con reacciones tuberculino negativas, scn vacunades y pasados a otra sección del establecimiento, hasta la aparición de las reacciones de alergia: en este momento $\operatorname{sen}$ reintegrados al medio o bien quedan por un tiempo más en el mismo establecimiento.

\section{Tiempo de aparición de la alergia.}

Ya hemos dicho que para comprobar si una persona está o no infectada de tuberculosis, se procede a las pruebas de reacciones a la tuberculina, tomando en cuenta que el períado de incubación o pre alérgico es de 6 a 7 semanas.

Las diversas experiencias de Wallgren. Rinvik. Dahl. Birckhaug, etc.. demuestran que la aparición de la alergia en los vacunados es muy variable y más que de la dosis de vacuna empleada. depende del método usado en las investigaciones de las reacciones a la tuberculina.

El profesor Andersen, en Dinamarca, ha investigado en 102 recién nacidos, vacunados por vía intra-dérmica y sub-cutánea, demostrando que a las tres o cuatro semanas es posible obtener reacciones positivas por los métodos de Hamburger y Moro.

El profesor Wallgren, en Suecia, quien emplea desde hace tiempo soluciones crecientes de Mantoux, que varían de $1 \times 10.000$ a $1 \times 10$, ha logrado establecer de un modo general que lo alergia tarda en aparecer entre 6 a 7 semanas.

Rinvik, en Noruega, siguiendo las experiencias del Prof. Wallgren, ha obtenido los mismos resultados en cuanto al tiempo de aparición de la alergia post-vaccinal. Sus porcen- 
tajes de positividad a la tuberculina después del octaro mes de vacunación, dan cifras más bajas que otros investigadores:

Birckhaug ha obtenido $98 \%$ de positivos.

Wallgren ha obtenido $90 \%$ de positivos.

Winge ha obtenido de 90 a $93 \%$ de positivos.

Rinvik ha obtenido de 74 a $75 \%$ de positivos.

La causa de ello se podría explicar en parte, por las condiciones de aislamiento, que en los casos estudiados por Rinvik han sido rigurosos, pero Wallgren, que ha hecho sus experiencias en igual forma, ha obtenido un porcentaje de positividad de $90 \%$, lo que Rinvik lo explica como que es más bien la técnica del Mantciux empleada por el profesor sueco, técnica que da una mayor rapidez de reacción que las pruebas percutáneas empleadas por él en algunos casos.

Duración de la alergia.

Muy estudiada y discutida ha sido la determinación del tiempo que dura la alergia provocada por la vacuna.

Se ba visto que las reacciones de la tuberculina son menos intensas en los vacunados, y que desaparecen en un tiempo que varía entre uno y cinco años. Es difícil establecer en los casos prolongądos de alergia hasta dónde puede ésta ser debida solamente a la vacuna, ya que el individuo devuelto al medio normal está cada dia expuesto a contagio. Experiencias hechas por Birckbaug, en animales. ban determinado un tiempo máximo de duración de la alergia de dos años.

Debido a estas divergencias es que se procede al control de los vacunados periódicamente cada año, tanto en sus reacciones tuberculínicas, como en exámenes médicos y de rayos.

Morbllidad y mortalidad tuberculosa en los vacnnados.

A pesar de que los términos de alergia e inmunidad tuberculosas están aún sometidos a discusión, ya que ambos fenómenos se presentan en la clínica simultáneamente, los resultados obtenidos en los vacunados y en los no vacunados frente a un foco de contagio, hablan de una disminución en la morbilidad y mortalidad en los primeros.

Enderson y Belfrage, en Gotemburgo, han seguido et control durante 10 años en 905 personas vacunadas y que. 
reintegradas al medio, ofrecian posibilidades de contagio. En el primer año después de la vacunación observaron 2 casos de pleuresia y un eritema nodoso, que evolucionaron favorablemente. Entre los vacunados restantes y en los 9 años siguientes no se presentó en ninguno de ellos algún caso de enfermedad o muerte por tuberculosis.

Wallgren, en 230 nin̄os vacunados y reintegrados a su medio, observó durante el primer año un solo caso comprobado de tuberculosis, cuya evolución fué benigna. De los 230, 2 murieron y la autopsia no reveló lesiones tuberculosas.

Las cifras que se han obtenido en la ciudad sueca de Gotemburgo, donde se han practicado la mayoría de las experiencias de vacunación, demuestran que la mortalidad por tuberculosis desde 1912 a 1933, en adultos y niños, son las siguientes:

\begin{tabular}{|c|c|c|}
\hline Año & $\begin{array}{l}\text { Mortalicad por } \\
10.000 \text { adultos }\end{array}$ & $\begin{array}{l}\text { Mortalidad por } \\
10,000 \text { nis̃os }\end{array}$ \\
\hline 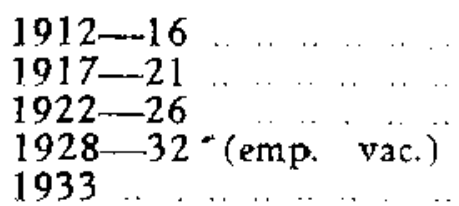 & $\begin{array}{l}20,7 \\
17,6 \\
13,5 \\
11,4 \\
10,0\end{array}$ & $\begin{array}{l}4,3 \\
4,2 \\
3,4 \\
1.4 \\
0,3\end{array}$ \\
\hline
\end{tabular}

De 15,000 reclutas vacunados por el B. C. G.. en Suecia, Lindsj observó que se presentaron 4 casos de tuberculosis primaria y 5 pleuresías, las que evolucionaton en forma benigna.

Rinvik, en Noruega, en sus observaciones: hechas en 2.103 niños no vacunados y 708 vacunados expuestos a contagio, comprobó que el $10,03 \%$ de los no vacunados hicieron una infección tubetculosa. mientras que en los vacunados la cifra fué $0,75 \%$, el tiempo de contagio demoró más en producirse y su evolución fué benigna. Respecto a la mortalidad observó $1,6 \%$ entre los no vacunados y $0 \%$ entre los vacunados.

Las experiencias de Scheel, en vacunaciones de los estudiantes de medicina, y las experiencias de Heimbeck con las alumnas de la Escuela de Enfermeras de Oslo, revelan que la morbilidad y la mortalidad tuberculosas son más elevadas en los sujetos insensibles a la taberculina, que en los alérgicos, y las curvas de morbilidad $y$ mortalidad en la 
alergia post-vaccinal son inferiores a las obtenidas en los alérgicos naturales.

Las experiencias citadas, y las practicadas en adultos en Francia (Troisier y Nico), y en niños en Canadá (Norbert Mezina), demuestran que, aun cuando no se han seguido las prácticas absolutas de aislamiento, las curvas de morbilidad $y$ mortalidad por tuberculosis son considerablemente menores en los vacunados por el B. C. G.

Dado los resultados obtenides, en Suecia se practica hoy en día la vacunación en todo recién nacido; es obligatoria para los estudiantes de medicina, alumnas de Escuelas de Enfermeras y reclutas tuberculino negativos. Los controles post-vaccinales son llevados en forma rigurosa.

En Noruega y Dinamarca la vacunación no se practica con tanta intensidad en los recién nacidos, salvo en los hijos de madres tuberculosas. En los escolares, aun cuando no es obligatorio. se ha cesseguido en los últimos años la vacunación de casi todos los alumnos que al término de la Escuela Primaria presentan aún tuberculinas negativas (igual cosa se hace en Suecia).

Las experiencias y buenos resultados obtenidos por esti método de prevención son numercsísimas y a través de esta exposición sólo se ha hecho mención de algunas de ellas. La desconfianza que existía en un principio, se ha transformado, ante los resultados obtenidos, en un verdadero entusiasmo por seguir en este camino, camino que para ncsctros, como médicos y como chilenos, debemos contemplar como un ejemplo.

Nuestro proceso evolutivo, cultural, político $y$ económico no es el mismo aue el de esos países nórdicos y debemos considertar, antes de actuar en una campaña antituberculosa. la realidad de nuestro medio.

Al exponer lo que pude comprobar personalmente en cuanto al resultado obtenido con el B. C. G. en Escandinavia, no me ha movido ctro objeto que el de demostrat que la utilidad de un descubrimiento científico es paralela a la calidad material y cultural del pueblo que ha de recibirla. 